Authors' reply: Professor Chaturvedi raises the possibility that our systematic review of the length of the duration of untreated psychosis in LAMI countries was confounded by a definition of treatment that was based on presentation to psychiatric services and did not account for presentations to traditional healers.

We acknowledge that a minority of the studies included in our review were based on population-based surveys of psychosis and that most of the studies did not include patients who only presented to traditional healers or did not receive any psychiatric treatment.

However, poor outcome in schizophrenia is known to be associated with delay in commencing treatment with antipsychotic medication, whereas little is known about the effects of a delay in non-pharmacological treatment. Furthermore, in a literature review (submitted for publication: details available on request) we confirmed the findings of Marshall et al ${ }^{1}$ that the adverse effects of delaying antipsychotic treatment are similar in high-income and LAMI countries. Hence, we believe that non-psychiatric treatment for psychosis is best thought of as a potential cause of prolonged duration of untreated psychosis, rather than a confounding factor in the definition of duration of untreated psychosis.

Psychoses with acute onset and short duration that might remit without treatment may be more common in LAMI countries. ${ }^{2,3}$ Patients with a short-lived psychosis might not always present to psychiatric services in LAMI countries, although in high-income countries acute psychosis is associated with a shorter duration of untreated psychosis. We do not know whether the exclusion of patients with a potentially short duration of untreated psychosis and those who only present to traditional healers would increase or decrease the mean period of nontreatment. In our review, population-based studies tended to report much longer mean periods of non-treatment than studies based on presentation to psychiatric services, although it is also possible that the lower mean duration of untreated psychosis found in upper-middle income countries was due to more individuals with an acute onset presenting for treatment early in their illness.

We agree that the pathways to care through non-psychiatric treatments warrant further investigation, but these studies should be conducted as part of an effort to reduce the unacceptably long duration of untreated psychosis in many LAMI countries.

1 Marshall M, Lewis S, Lockwood A, Drake R, Jones P, Croudace T. Association between duration of untreated psychosis and outcome in cohorts of first episode patients. Arch Gen Psychiatry 2005; 62: 975-83.

2 Susser E, Wanderling J. Epidemiology of nonaffective acute remitting psychosis vs schizophrenia. Sex and sociocultural setting. Arch Gen Psychiatry 1994; 51: 294-301

3 Mojtabai R, Susser E, Varma V. Duration of remitting psychoses with acute onset. Implications for ICD-10. Br J Psychiatry 2000; 176: 576-80.

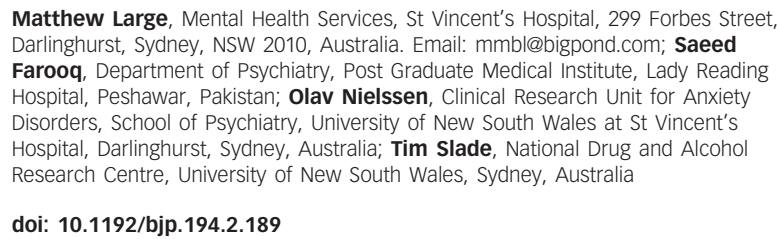

doi: 10.1192/bjp.194.2.189

\section{To prescribe or not to prescribe?}

Despite the possible heterogeneity among some of the studies included in Tsapakis et al's study, ${ }^{1}$ the results, if accepted by the psychiatric fraternity, could lead to further reduction in the use of antidepressants in the child and adolescent population. The use of antidepressants in this group has already decreased by
33\% since the Committee on Safety of Medicine's (CSM's) warning against the use of most antidepressants in children and adolescents. ${ }^{2}$ Although the National Institute for Health and Clinical Excellence guidelines on the treatment of depression among children and adolescents states that medication should only be used in conjunction with psychological interventions, ${ }^{3}$ the provision of psychological therapies remain thin on the ground in most parts of the country, which means that medication is often the only option available to clinicians for treatment of severe depression.

Although purely pharmacological treatment would be the least desirable option in depression and research evidence on the efficacy of antidepressants for those with depression in all age groups is either mixed or at best shaky, depending on which side of the debate one is on, ${ }^{4}$ most clinicians would agree that many patients with significant depression do improve on antidepressants. Although it is too early to judge whether reduction in antidepressant prescribing resulting from the CSM warning has resulted in an increase in depressive morbidity among children and adolescents in the UK, disturbing evidence is already emerging from the USA, Canada and The Netherlands ${ }^{5}$ on an increase in completed suicide among children and adolescents, which seems to coincide with the reduction in antidepressant prescribing following warnings by regulatory agencies. In a retrospective study done in Canada, a significant reduction in antidepressant prescribing, accompanied by a statistically significant increase in suicide among children and adolescents (relative risk $=1.25$, 95\% CI 1.08-1.44; annual rate per $1000=0.04$ before and 0.15 after the warning) was noted in the 2 years following issuance of the warning. ${ }^{6}$

Given the well-established link between depression and suicide, one can only conclude that clinicians may be undertreating depression in children and adolescents since the emergence of concerns in relation to antidepressants. I feel clinicians should use their own clinical judgement and take into account local resources before making decisions on the course of treatment in juvenile depression. This would help one maintain the right balance between evidence-based practice and what's best for individual patients, especially in an area of practice where research evidence is often ambiguous and contradictory.

1 Tsapakis EM, Soldani F, Tondo L, Baldessarini RJ. Efficacy of antidepressants in juvenile depression: meta-analysis. Br J Psychiatry 2008; 193: 10-7.

2 Kurian BT, Ray WA, Arbogast PD, Fuchs DC, Dudley JA, Cooper WO. Effect of regulatory warnings on antidepressant prescribing in children and adolescents. Arch Pediatr Adolesc Med 2007; 161: 690-6.

3 National Collaborating Centre for Mental Health. Depression in Children and Young People. British Psychological Society \& Royal College of Psychiatrists, 2005.

4 Moncrieff J, Kirsch I. Efficacy of antidepressants in adults. BMJ 2005; 331: 155-7.

5 Yan J. Canada sees troubling trend in antidepressant prescribing. Psychiatr News 2008; 43: 14

6 Katz LY, Kozyrskyj AL, Prior HJ, Enns MW, Cox BJ, Sareen J. Effect of regulatory warnings on antidepressant prescription rates, use of health services and outcomes among children, adolescents and young adults. CMAJ 2008; 178: 1005-11.

Krishna Menon, Greenwich and Bexley CAMHS, Highpoint House, Shooters Hill, London SE18 3RZ, UK. Email: kmenon@nhs.net

doi: 10.1192/bjp.194.2.189a

Authors' reply: We agree with Menon that, in clinical practice, many juvenile patients with depression almost certainly are underdiagnosed, reluctant to accept treatment, undertreated or leave treatment prematurely, and that competent clinical help, especially other than the use of antidepressants, for such patients and their 
families is hard to find. However, the proposition that antidepressants may have similar effects at all ages is inconsistent with our findings of quite limited, and perhaps inversely age-dependent, efficacy of antidepressants, as a class, as well as a lack of statistically significant differences between older and modern agents (especially of tricyclics $v$. serotonin reuptake inhibitors), and the powerful influence of study size on conclusions about 'significance' of separation of antidepressants from placebos. ${ }^{1}$

A timely and pressing question is whether antidepressant treatment alters suicidal risks. Depression and suicide are strongly associated, but prediction of suicidal behaviour, even in individuals with depression, is very difficult, and evidence concerning relationships of antidepressant treatment to suicidal behaviour, although consistent in randomised clinical trials, remains controversial. ${ }^{2,3}$ Whether or not youth suicide rates will consistently increase or decrease, remains to be seen, and to be sorted out from high international variation in yearly suicide rates and poor documentation of attempts. ${ }^{2}$

For now, it seems an inescapable conclusion that clinicians are left to their own clinical judgement about using antidepressants for young individuals diagnosed with major depressive disorder. Furthermore, disbelief that modern antidepressants show relatively modest effects compared with placebos and fail to separate statistically from older agents, ${ }^{1}$ paired with the repeated and the poorly documented assertion that some modern antidepressants work well in clinical practice, seems to avoid the issues. We considered various ways in which even randomised controlled trials may be misleading, including selection of atypical or mildly ill out-patients or use of inadequate doses of antidepressants, ${ }^{1}$ as well as current controversy about how to diagnose and quantify changes in affective disorders in children and adolescents. ${ }^{4}$ Nevertheless, it is difficult to simply dismiss and ignore the findings of the research that has been done to test the efficacy of antidepressants in juvenile depression. ${ }^{1}$

1 Tsapakis EM, Soldani F, Tondo L, Baldessarini RJ. Efficacy of antidepressants in juvenile depression: meta-analysis. Br J Psychiatry 2008; 193: 10-7.

2 Baldessarini RJ, Tondo L, Strombom I, Dominguez S, Fawcett J, Oquendo M, et al. Analysis of ecological studies of relationships between antidepressant utilization and suicidal risk. Harv Rev Psychiatry 2007: 15: 133-45.

3 Hammad TA, Laughren T, Racoosin J. Suicidality in pediatric patients treated with antidepressant drugs. Arch Gen Psychiatry 2006; 63: 332-3.

4 Henry C, Demotes-Mainard J. SSRIS, suicide and violent behavior: is there need for a better definition of the depressive state? Curr Drug Saf 2006; 1: 59-62.

\footnotetext{
Evangelia M. Tsapakis, Department of Psychological Medicine and MRC Social, Genetic and Developmental Psychiatry Centre, Institute of Psychiatry, King's College London, UK. Email: e.tsapakis@iop.kcl.ac.uk; Federico Soldani, Department of Epidemiology, Harvard School of Public Health, and Department of Psychiatry, Harvard Medical School and Psychopharmacology Program, McLean Division of Massachusetts General Hospital, Boston, Massachusetts, USA; Leonardo Tondo, ucio Bini, Mood Disorder Centre and Department of Psychology University of Lacio Bini, Mood Disorder Psychiatry, Harvard Medical School, Psychopharmacology Program and International Consortium for Bipolar Disorder Research, McLean Division of Massachusetts General Hospital, Boston, Massachusetts, USA

doi: 10.1192/bjp.194.2.189b
}

\section{Effects of an earthquake on suicide rates in Nantou, Taiwan}

The massive earthquake in Sichuan, China, that occurred on 12 May 2008 left 92000 dead or missing, almost 374000 injured, and millions homeless.

Rebuilding the communities is a huge task and much is to be learnt from communities with similar experiences. On 21 September 1999, Nantou County in Taiwan experienced an earthquake measuring 7.3 on the Richter scale. It caused more than 2000 deaths, 10000 injuries and 100000 collapsed buildings. ${ }^{1}$
After the earthquake, the number of suicides surged in Nantou. ${ }^{1,2,3}$ The general patterns of suicide in both regions are similar; ${ }^{4,5}$ what happened in Nantou after the earthquake should inform suicide prevention in Sichuan.

Table 1 shows the suicide rates in Nantou before and after 1999. The female suicide rate more than doubled immediately from 6.1 in 1998 to 14.2 in 1999 , whereas a very small increase was observed in males. However, the male suicide rate showed substantial increases in both 2000 and 2001, indicating a delayed effect. On the whole, the rate of increase in Nantou was higher than that in other parts of Taiwan (81\% v. 25\%).

\begin{tabular}{|c|c|c|c|c|c|c|}
\hline & \multicolumn{2}{|c|}{ Male } & \multicolumn{2}{|c|}{ Female } & \multicolumn{2}{|c|}{ Overall } \\
\hline & Nantou & Taiwan & Nantou & Taiwan & Nantou & Taiwan \\
\hline \multicolumn{7}{|c|}{ Suicide rate (per 100 000) } \\
\hline 1998 & 18.6 & 12.8 & 6.1 & 6.9 & 12.6 & 9.9 \\
\hline 1999 & 19.0 & 13.7 & 14.2 & 6.8 & 16.7 & 10.3 \\
\hline 2000 & 23.7 & 14.4 & 13.1 & 7.6 & 18.7 & 11.1 \\
\hline 2001 & 33.6 & 16.6 & 11.2 & 8.0 & 22.9 & 12.4 \\
\hline \multicolumn{7}{|c|}{$\begin{array}{l}\text { Change when compared } \\
\text { with } 1998, \%\end{array}$} \\
\hline 1999 v. 1998 & 2.32 & 6.64 & 131.92 & -1.01 & 32.36 & 3.93 \\
\hline 2000 v. 1998 & 27.57 & 12.81 & 114.01 & 9.84 & 47.55 & 11.68 \\
\hline 2001 v. 1998 & 80.99 & 29.77 & 82.25 & 16.21 & 81.09 & 24.97 \\
\hline
\end{tabular}

The death of one's spouse may trigger suicidal thoughts, especially when compounded with the loss of the major income source. As men are more likely to be the 'bread winner' in rural areas, widows might suffer from a profound feeling of hopelessness immediately after a natural disaster. In the case of Sichuan, it is further aggravated by the loss of many children in the collapsed schools, many of them from one-child families (owing to the State's family policy). In contrast, unemployment carries major risk for male suicides; men are likely to be of high risk when the earthquake's impact on the local economy is fully manifested. This can explain the gender difference in the timing of heightened suicide risk in Nantou. It also suggests that the restoration efforts in Sichuan should devote resources to preventing suicide attempts among women in the short term, while devising strategies to prevent further causalities for male suicides before the local economy fully recovers.

\section{Acknowledgements}

The author would like to pay tribute to those who have worked tirelessly to save the survivors.

1 Han SN, Chen KT. Mortality of the 921 earthquake in Nantou and Taichung counties. Epidemiol Bull 2000; 1: 1-8.

2 Liaw YP, Wang PW, Huang CC, Chang CM, Lee WC. The suicide mortality rates between 1997-1998 and 2000-2001 in Nantou County of Taiwan following the earthquake of September 21 in 1999. J Forensic Sci 2008; 53: 199-202.

3 Chou YJ, Huang N, Lee CH, Tsai SL, Tsay JH, Chen LS. Suicides after the 1999 Taiwan earthquake. Int J Epidemiol 2003; 32: 1007-14.

4 Liu KY, Beautrais A, Caine E, Chan C, Chao A, Conwell Y, et al. Charcoal burning suicides in Hong Kong and urban Taiwan: an illustration of the impact of a novel suicide method on overall regional rates. $J$ Epidemiol Community Health 2007: 61: 248-53.

5 Yip $\mathrm{P}$, Liu $\mathrm{K}$, Song XM, Hu JP. Suicide rate in China during a decade of rapid social changes. Soc Psychiatr Psychiatr Epidemiol 2005; 40: 792-8.

Paul Yip, Social Work and Social Administration, Centre for Suicide Research and Prevention, The University of Hong Kong, Hong Kong. Email: sfpyip@hku.hk

doi: 10.1192/bjp.194.2.190 